\title{
Surgical management of brain metastases
}

\author{
Moksha G. Ranasinghe, M.D., AND Jonas M. Sheehan, M.D. \\ Department of Neurosurgery, Pennsylvania State University, Hershey, Pennsylvania
}

\begin{abstract}
$\checkmark$ Metastatic brain tumors continue to increase in incidence as patients with cancer live longer. The options for management continue to evolve as well, with advances in radiation-based treatment, chemotherapy, and surgery. Although metastatic brain tumors are frequently treated without surgical intervention, there continues to be a significant role for surgery in caring for patients with these lesions. Study data have proven that surgery has a positive effect on survival and quality of life in properly selected patients. Those with a suitable age, functional status, systemic disease control, and several metastases may be suitable for surgical treatment. Advances in preoperative imaging and planning as well as intraoperative surgical adjuncts have lowered the morbidity associated with resection. With proper patient selection and operative and postoperative management, resection continues to play a significant and evolving role in the care of patients with metastatic brain tumor.
\end{abstract}

KEY WORDS • brain metastases - surgery - radiosurgery - whole-brain radiotherapy

$\mathrm{M}$ ETASTATIC brain tumors are diagnosed in nearly 150,000 patients annually in the US, arising in 10 to $40 \%$ of patients with cancer. ${ }^{15}$ The most common primary sources of brain metastases are lung $(17 \%)$, renal cell $(10.5 \%)$, and breast $(5.2 \%)$ cancer and melanoma $(8 \%){ }^{31}$ With the increase in the early detection of primary tumors and longer survival in patients with cancer, the incidence of brain metastases is rising. Improvements in imaging quality and accessibility have also contributed to the increased number of patients in whom metastasis is diagnosed. Modern imaging technology can detect smaller lesions, allowing for earlier intervention than was possible in the past and perhaps increasing the opportunity to control CNS disease. The majority of patients who have local CNS tumor control die of extracranial disease progression, whereas those with uncontrolled brain metastases more often die of neurological causes. ${ }^{2}$ Therefore, achieving local control is of primary importance when considering treatment options in patients with brain metastases. ${ }^{42}$

Because of its less invasive nature and apparent effec-

Abbreviations used in this paper: $\mathrm{BCNU}=1,3$-bis(2-chloroethyl)-1-nitrosurea; CNS = central nervous system; $\mathrm{CT}=$ computed tomography; KPS = Karnofsky Performance Scale; MR = magnetic resonance; RPA = recursive partitioning analysis; RTOG = Radiation Therapy Oncology Group; WBRT = whole-brain radiotherapy. tiveness for metastatic disease, stereotactic radiosurgery is chosen over resection with increasing frequency. However, surgical removal is a mainstay in the treatment of brain metastases. Advances in surgical techniques permit resection with decreasing morbidity, thus making resectable certain metastases that were previously considered unresectable. Such advances improved surgical access to metastases and allowed more aggressive resections with correspondingly low surgical complications and morbidity.

\section{Role OF SURGERY}

The importance of surgery for local control in brain metastases has been confirmed during the past few decades. Previously, WBRT was the mainstay in the treatment of intracranial metastases. Authors of several randomized trials have validated the benefit of surgery. ${ }^{25,39} \mathrm{In}$ 1990 Patchell and colleagues ${ }^{25}$ randomized 48 patients with single brain metastases to surgery and WBRT (25 patients) compared with WBRT alone (23 patients) and evaluated local recurrence and survival rates. In this study, the addition of surgery reduced the local recurrence in these patients from 52 to $20 \%(\mathrm{p}<0.02)$ and improved median survival from 15 to 40 weeks $(p<0.001)$. Patients in the surgery combined with WBRT arm also remained functionally independent for a longer period of time than those treated with WBRT alone (38 weeks compared with 8 weeks; $p<0.005)$. This result demonstrated the importance of resection in patients with single metastases. ${ }^{39}$ 
TABLE 1

Comparison of median survival times among patients with metastatic brain disease according to type of treatment*

\begin{tabular}{ccccccc}
\hline \hline & & & \multicolumn{3}{c}{ Months of Survival } \\
\cline { 5 - 7 } $\begin{array}{c}\text { Authors } \\
\text { \& Year }\end{array}$ & $\begin{array}{c}\text { No. of } \\
\text { Patients }\end{array}$ & $\begin{array}{c}\text { Primary } \\
\text { Tumor Type }\end{array}$ & $\begin{array}{c}\text { Supportive } \\
\text { Care }\end{array}$ & Radiotherapy & Surgery & $\begin{array}{c}\text { Surgery+ Radio- } \\
\text { therapy }\end{array}$ \\
\hline $\begin{array}{l}\text { Sampson et al., 1998 } \\
\text { Largerwaard et al., 1999 }\end{array}$ & 702 & melanoma & NA & 4.0 & 6.5 & 8.7 \\
Fife et al., 2004 & 1292 & $\begin{array}{c}\text { lung, breast, \& } \\
\text { melanoma }\end{array}$ & 1.3 & 3.6 & NA & 8.9 \\
\hline
\end{tabular}

$*$ NA $=$ not applicable.

In 1998 Patchell and colleagues ${ }^{24}$ investigated the benefit of using WBRT as an adjunctive therapy following surgical tumor removal in these patients. The study randomized 95 patients to surgery alone or surgery plus WBRT. Progression of intracranial disease was fourfold greater in the surgery-alone group (70\% compared with $18 \%, \mathrm{p}<0.001)$, and local recurrence was also higher in this group (46\% compared with $10 \%, \mathrm{p}<0.001$ ). Patients treated with surgery alone received WBRT at the time of CNS disease progression, and so survival data did not reflect the initial randomization of the groups. There was comparable survival between the groups: surgery plus WBRT, median survival 12.0 months; and surgery alone, median survival 10.8 months. These data support the use of WBRT with surgery for improvement in recurrence and local control.

\section{Prognosis of Brain Metastasis}

The overall prognosis of brain metastasis has improved over the past two decades with the use of combined therapy. Table 1 shows a comparison of median survival rates in patients with brain metastases from three large studies in which the authors compared supportive care with radiotherapy alone, surgery alone, and surgery plus WBRT. ${ }^{34}$ The first two studies ${ }^{13,29}$ included patients with cerebral metastases from melanoma, and the third study included patients with brain metastases from lung, breast, and melanoma primary tumors. Together, the studies included 3131 patients, and treatment was stratified according to the number of brain metastases, presence and control of extracerebral metastases, and performance status. Regardless of the histological features of the primary tumor, patients who received supportive therapy had median survival times of 1 to 2 months. Radiotherapy improved survival by 3 to 4 months in these patients. Among patients with a single metastasis, those who underwent resection had a 7- to 9-month increase in mean survival, with a modest improvement beyond this point with the addition of radiation therapy. Data from these retrospective studies highlight the importance of treatment and control of local disease with the use of surgery alone or combined with radiotherapy.

\section{Radiosurgical Treatment}

Radiosurgery has been used for more than 30 years as an alternate treatment to surgery, initially mostly in benign tumors such as meningiomas and vestibular schwanno- mas. In the past 15 years, it has emerged as an effective and attractive modality for the treatment of brain metastases. The rationale for using radiosurgery rather than WBRT is that a high focal dose of radiation can improve local tumor control while avoiding the potential toxicities of WBRT. Radiosurgery-induced tumor control can be achieved by early effects (apoptosis or mitotic death) or by delayed vascular changes or immune responses. ${ }^{22} \mathrm{Re}-$ sults from several studies have shown that metastases that are resistant to WBRT (for example, sarcoma, melanoma, and renal cell carcinoma) can be treated with radiosurgery with control rates similar to those for tumors not considered radioresistant. $8,20,28,32,33$

In a multiinstitutional retrospective study, Auchter and colleagues $^{3}$ reviewed treatment and survival in 122 patients with resectable single brain metastases that had been treated with radiosurgery and WBRT; these patients were compared with matched cohorts from resection and WBRT arms of randomized controlled trials. The median survival in patients who received radiosurgery was 56 weeks, the local tumor control rate was $86 \%$, and the duration of functional independence (KPS score > 70) was 44 weeks. The local control rate and duration of functional independence were comparable with those in the resection arm of Patchell and colleagues' study:25 median survival of 40 weeks, local control rate of $80 \%$, and duration of functional independence of 38 weeks. Therefore, radiosurgery plus WBRT yielded results similar to those achieved with surgery plus WBRT while avoiding the risks, discomfort, and recovery periods associated with surgery.

Radiosurgery offers some advantages for the treatment of brain metastases (Table 2). It is possible in a single session to treat several metastases by precisely localizing them with the aid of MR imaging or CT scanning. Many brain metastases are small, well-circumscribed spherical lesions found at the gray-white junction. Treatment of brain metastases as distinct targets, without intervening healthy brain tissue, allows delivery of high-dose radiation to the discrete target volume with a sharp falloff at the boundary so that the adjacent normal brain tissue receives a relatively safe dose of radiation. This approach allows delivery of a high dose of radiation to the lesion in a single fraction, something not achieved with WBRT. An additional benefit of radiosurgery is its minimally invasive nature. It can be performed on an outpatient basis and in a single session, with local anesthesia eliminating the need for hospitalization or multiple treatments.

There are some limitations and disadvantages to the use 
TABLE 2

Comparison of surgery and radiosurgery for the treatment of patients with brain metastases

\begin{tabular}{|c|c|}
\hline Surgery & Radiosurgery \\
\hline \multicolumn{2}{|l|}{ advantages } \\
\hline allows histological diagnosis & minimally invasive \\
\hline removes mass effect & no hospitalization \\
\hline improves local control & cost effective \\
\hline treatment of recurrence & treatment of recurrence \\
\hline able to treat large lesions & treats surgically inaccessible masses \\
\hline \multicolumn{2}{|l|}{ disadvantages } \\
\hline invasive & no histological diagnosis \\
\hline requires hospitalization & limited to small tumors \\
\hline limited to $1-3$ metastases & limited to $1-3$ metastases \\
\hline infection & longer time for resolution of mass effect \\
\hline
\end{tabular}

of radiosurgery for brain metastases. No histological diagnosis is illuminated with radiosurgery as there is with resection. Large tumors (typically those $>3 \mathrm{~cm}$ in diameter) may not be appropriate for radiosurgery. Furthermore, among patients symptomatic due to mass effect, the lack of relief from mass effect after radiosurgery makes the shortterm survival in these patients even more brief than if they had been treated with resection. Moreover, the potential for increased edema is a concern after radiosurgery, although in many cases tumor death leads to a decrease in edema during the first few weeks after treatment.

\section{Selecting For Resection}

\section{Patient Age, Functional Status, and Extracranial Disease}

Careful selection of patients to undergo resection is important given that surgery is not likely to benefit all patients with cerebral metastases (Table 3). The patient must be medically suitable for both surgery and recovery before proceeding with resection, and the disease prognosis should be amenable to benefit from local CNS tumor control. The RTOG has developed an RPA to classify patients statistically by using KPS score, patient age, and status of extracranial disease. ${ }^{1}$ These guidelines were developed

TABLE 3

Factors favorable for tumor resection

patient age $<65$ yrs

KPS score $>70$

single tumor

tumor size $>3 \mathrm{~cm}$

surgical accessibility

good tumor localization

control of extracranial disease

absence of leptomeningeal involvement

undiagnosed primary site of cancer

long disease-free survival

local symptomatic mass effect based on a database of 1200 patients on which an RPA was performed to develop three prognostic classes for patients with multiple brain metastases (note that all patients had an age younger than 65 years, controlled primary disease, and no extracranial metastases): 1) RPA Class I: a KPS score greater than 70 (median survival 7.1 months); 2) RPA Class III: a KPS score less than 70 (median survival 2.3 months); and 3) RPA Class II: all others (median survival 4.2 months). ${ }^{14}$ The RTOG database was built from three previous RTOG Phase I/III trials predominantly made up of patients with multiple unresected brain metastases treated with WBRT. In these patients, age, KPS score, and systemic disease were important prognostic factors. Recursive partitioning analysis Class I patients are good candidates for craniotomy and resection, whereas Class III patients are not likely to realize benefit from surgery.

In a large single-institution retrospective study, Lagerwaard et al. ${ }^{19}$ reviewed prognostic factors in 1292 patients with CT-diagnosed brain metastases. Age, sex, performance status, number and distribution of metastases, site of primary tumor, histological features, interval between primary tumor occurrence and brain metastases, systemic tumor activity, serum lactate dehydrogenase level, response to steroid treatment, and treatment modality were evaluated in these patients. The median survival was 3.4 months, with 6-month, 1-year, and 2-year survival percentages of 36,12 , and $4 \%$, respectively. Survival was also statistically significantly different among the various treatment modalities, with a median survival of 1.3 months in patients receiving steroids alone, 3.6 months in those receiving radiotherapy, and 8.9 months in those undergoing neurosurgery followed by radiotherapy. Univariate and multivariate analyses were performed to determine significant prognostic factors. Preoperative performance status, symptomatic response to steroid treatment, systemic tumor control, and serum lactate dehydrogenase levels were independent prognostic factors. In patients with primary lung cancer, patient sex was an important prognostic factor as was the interval between primary tumor occurrence and the development of brain metastases in breast cancer.

\section{Tumor Grade and Histological Characteristics}

Surgery is often favorable in patients when the histological type of the brain metastasis is unknown and no likely source is identified on staging workup. Tumor histopathology may be important in developing a treatment plan for an individual with brain metastasis given that different histological types have different chemotherapeutic and radiation management options. ${ }^{6,18}$ Tumors such as sarcoma, renal cell carcinoma, and melanoma are considered resistant to WBRT. It has been shown that these tumors respond to radiosurgery as well as more "favorable" histologies. ${ }^{8,28,32,33}$ Staging of the tumor with size, location, and metastases and grading of the tumor according to histological findings both allow one to estimate prognosis. For surgery to afford a survival benefit, the life expectancy based on systemic disease should be greater than 3 months. With the advent of new therapies for systemic disease, the long-term survival in patients with cancer, such as those with breast cancer, has improved. Data from several studies have shown that, with surgery plus postsurgical radiotherapy, breast cancer has the best prognosis, 
whereas melanoma and renal cell cancer have the worst. ${ }^{29,33,45}$

\section{Single and Multiple Brain Metastases}

Since the 1990 study by Patchell and colleagues, surgery for single brain metastases has been the mainstay of treatment. At that time, the presence of multiple brain metastases frequently excluded a patient from surgical consideration because the patient was not expected to survive long enough to realize a benefit from surgery and because adequate resection of tumors was not expected. With improvements in surgical techniques, some lesions that were previously inaccessible are now considered accessible. Pollock and associates ${ }^{26}$ reviewed data from 52 patients who had undergone radiosurgery, tumor resection, or both to determine whether properly selected patients with multiple brain metastases could benefit from aggressive treatment of intracranial disease. The median age was 58 years, median KPS score was 90, and median number of tumors was three. On follow up, 20 patients $(38 \%)$ had tumor progression following radiation, and treatment included resection in $10 \%$ (five patients), radiosurgery in $60 \%$ (31 patients), and both in 30\% (16 patients). The median survival was 15.5 months. Results of multivariate analysis showed that RTOG RPA Class I correlated with improved survival, with patients surviving a median of 19 months. In contrast, patients in Class III survived a mean of 8 months and those in Class II survived a mean of 13 months. Multivariate analysis findings also revealed that patients with radiosensitive tumors (such as those from lung and breast primary sites) had fewer intracranial recurrences compared with patients harboring radioresistant tumors (for example, melanoma, renal, and sarcoma; relative risk $2.43,95 \%$ confidence interval $1.13-$ $5.10, \mathrm{p}=0.02$ ). Authors in this study supported aggressive treatment in RPA Class I and II patients with controlled primary disease and a limited number of metastases. In another study, Bindal et al. ${ }^{5}$ performed a retrospective review of 56 patients who had undergone resection of multiple brain metastases. Of these patients, $30 \mathrm{had}$ one or more lesions left unresected (Group A), and 26 underwent resection of all lesions (Group B). Another 26 patients with single metastasis (Group C) were selected to match Group B. The median survival in Group A was 6 months; in Groups B and C, 14 months. Results of statistical analysis showed a significant difference in survival in patients in Group A but not between those in Groups B and C. Similar results were found with regard to local recurrence and symptom relief following surgery. Data in this study demonstrated that it is possible with the use of resection to achieve an improved prognosis in patients with multiple brain tumors. Patients with four or more brain tumors are usually not treated surgically, given the poor prognosis in this situation.

\section{Other Tumor Characteristics Warranting Surgery}

Tumor location and size are important in planning treatment in a patient with brain metastases. If there is significant mass effect or if the tumor is indirectly affecting a region of eloquence, the patient may benefit from resection to alleviate mass effect for symptom relief and improvement in quality of life. If a patient has multiple

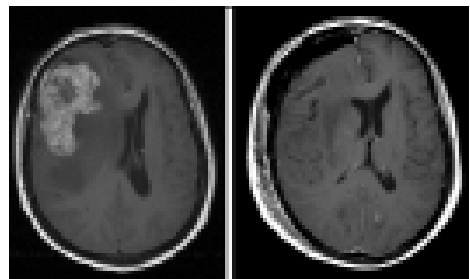

FIG. 1. Preoperative (left) and postoperative (right) images obtained in a 58-year-old woman with a known history of nonsmall cell lung cancer who had presented with left-sided weakness, demonstrating the status of a large dominant frontal lesion. The patient elected to undergo resection for relief of mass effect and confirmation of diagnosis.

lesions and one dominant lesion causing mass effect that is life threatening or reducing the quality of life, resection of the dominant lesion is preferable. Larger metastases $(>3 \mathrm{~cm})$ that are surgically accessible are better treated with resection than with WBRT or radiosurgery alone (Fig. 1). Medically uncontrollable seizures due to brain metastasis are an indication for resection, as surgery can provide seizure relief.

\section{Leptomeningeal Disease}

Leptomeningeal carcinomatosis involves diffuse infiltration of leptomeninges by cancer cells and most commonly occurs with lung and breast cancers. The presence of leptomeningeal disease is associated with a poor prognosis. Surgery has not been shown to provide significant benefit in individuals with this disease, and the mainstay in treatment is radiation and intrathecal or systemic chemotherapy. ${ }^{36}$ Authors of several studies have shown that the absence of meningeal carcinomatosis on diagnosis is an independent predictor of prolonged survival. ${ }^{45}$

\section{Recurrent Metastases}

Surgery has been shown to improve survival and quality of life in patients with recurrent disease. ${ }^{2,4}$ Resection of recurrent tumor also allows confirmation of histopathology and the use of local chemotherapeutic adjuncts such as BCNU wafer implants. It is important to distinguish between actual recurrence and necrosis to plan adjuvant therapy, because an accurate diagnosis based solely on imaging can be difficult.

\section{TeChniques And Goals of Surgery}

Preoperatively, patients should be screened so that surgery is considered in those who are likely to benefit from resection. Patients with a younger age, high KPS score, and limited extracranial disease (RPA Class I) are favorable candidates. Tumor histopathology factors into the determination of the optimal treatment modality, as some tumors are more radiosensitive than others. The number and location of metastases are also important as prognostic markers and affect treatment planning. Contrast or double-contrast MR imaging is more sensitive in detecting small lesions than contrast CT. Computed tomography studies have shown single lesions in up to $50 \%$ of patients, with approximately $20 \%$ harboring just two lesions. ${ }^{9}$ Magnetic resonance imaging studies reveal 
that only approximately 25 to $33 \%$ of patients have truly solitary lesions. ${ }^{35}$ Therefore, patients with single lesions on CT scanning may have multiple lesions on MR imaging and should be evaluated with the latter procedure before surgery if possible. It is also important to evaluate all patients considered for surgery medically by obtaining a thorough history and performing a physical examination and preanesthesia evaluation to determine whether the patient has an acceptable risk for surgery. Preoperative steroids (mostly dexamethasone) are given to decrease cerebral edema and for symptomatic relief. The administration of such agents may also help the surgeon to distinguish preoperatively deficits related to the tumor as opposed to those arising from peritumoral edema.

In most cases, the primary goal of surgery is gross-total resection of the tumor with minimal disruption of, or injury to, the brain. In some cases, the additional goals of debulking and relief of mass effect are considered. Data from a retrospective review performed by Korinth et al. ${ }^{23}$ in 2002 in patients who had undergone microsurgical tumor removal between 1989 and 1996 showed that the early postoperative KPS score was improved in 59\% of patients, unchanged in 32\%, and worse in 9\%. Results in this study indicate that function is greatly improved, if not preserved, with microsurgical resection. Surgery also allows for histological confirmation of the diagnosis of the brain metastases.

Techniques of resection have greatly improved with the advances in preoperative imaging and image-guided surgery. In fact, image-guided surgery has become routinely used in elective resection of metastatic tumors. ${ }^{10,21,37,43}$ Frameless stereotactic guidance has become a standard procedure in most neurosurgical centers. This approach allows for accurate localization of the tumor before surgery to reduce the required craniotomy size, to avert injury to normal brain tissue, and to minimize searching for the tumor during surgery. Many metastatic lesions have a clear textural difference compared with healthy brain tissue, which facilitates dissection of the tumor, but some lesions have indistinct borders. In cases of resection and tumors with cystic components, significant brain shift can occur; in such situations 2D ultrasonography can be helpful in determining the location of tumor, adjacent structures, cystic regions, and margins. ${ }^{38}$

Functional MR imaging and electrocortical mapping can also aid in delineating eloquent areas in the brain both preoperatively and intraoperatively. ${ }^{41}$ Functional MR imaging maps can be matched and fused with high-resolution MR or CT images to produce neuronavigational images, a process referred to as "functional neuronavigation." No randomized trials or outcome studies have been performed to show the patient benefits of applying preoperative functional MR imaging. The gold standard for mapping remains intraoperative cortical mapping. Using this method of monitoring, it is possible to resect spaceoccupying metastases in the sensorimotor cortex ${ }^{10}$ while minimizing deficits. Direct bipolar electrical stimulation of exposed cortex for motor cortex mapping offers more functional information than that obtained through monitoring of cortical somatosensory evoked potentials and phase reversal phenomena. It is also possible to stimulate the white matter tracts directly during resection to monitor the location of critical fiber pathways.
During resection of tumor, one should avoid injury to or disruption of en passage vessels coursing through areas near the tumor and likely perfusing normal brain tissue, although this type of blood vessel is not typically seen. Vessels that are induced by and supplying the tumor can be coagulated and divided.

\section{Adjuncts to ReSEction}

In 1998 Patchell and colleagues ${ }^{24}$ demonstrated that there is a decrease in the recurrence of intracranial disease in patients treated with WBRT following resection. Data in this study did not demonstrate a definite improvement in survival, although the study was not designed to do so given the crossover of patients from the surgery-alone treatment to (surgery plus) WBRT after the detection of disease progression. Thus, for the control of local disease, it would be beneficial to follow resection with WBRT. This strategy is especially advocated in patients who are unable to undergo enhanced MR imaging following surgery to determine the intracranial tumor burden.

There is some anecdotal evidence that stereotactic radiosurgery applied to the bed of a resected tumor lowers local recurrence. ${ }^{12}$ Currently, there are no published data on the effects of radiosurgery following resection of brain metastases. Localized adjuncts such as BCNU wafer implants (Gliadel; Guilford Pharmaceuticals, Inc.) have been used to treat patients undergoing resection of newly diagnosed and recurrent brain metastases. Ninety-three patients with brain metastases (new and recurrent) were studied by three groups. ${ }^{7,11,17}$ In these three studies, 66 patients received WBRT following resection and wafer placement, ${ }^{7,11}$ and 27 patients in the PROLONG study received no WBRT. ${ }^{17}$ During the follow up, there was no local recurrence reported in 91 patients and only two neurologically related deaths. Therefore, data in these studies show that there may be a benefit for local chemotherapy with Gliadel.

\section{Postoperative Care}

A contrast-enhanced MR image is obtained, when possible, within 24 to 48 hours of surgery to confirm grosstotal tumor resection. General postoperative care with early mobilization assists in transitioning the patient to rehabilitation care early. Another problem in patients is the presence of peritumoral or postoperative edema. Corticosteroids are effective in reducing the degree of peritumoral vasogenic edema and are usually started preoperatively. Despite the advantages of using steroids acutely, corticosteroids have several undesired effects postoperatively: predisposing to poor wound healing, worsening of glucose control, and immune compromise. Therefore, it is important to wean patients from steroids as early as can be tolerated postoperatively, while considering other metastases that are present, to assist in optimizing overall surgical outcome..$^{27}$

Venous thromboembolic disease is a common problem in patients with cancer, occurring in approximately $15 \%$. Patients with metastatic brain tumors appear to be more prone to this complication, probably because they are more likely to be hemiparetic or bedridden. ${ }^{30}$ Postoperatively, the maintenance of pneumatic compression boots 
and compression stockings can help reduce the development of deep venous thromboses. Thereafter, one should consider early mobilization of the patient or long-term treatment with an inferior vena cava filter or low-dose heparin for deep vein thrombosis prophylaxis in the immobile patient.

Seizures are another problem afflicting patients with intracranial metastases. This fact does not justify the prophylactic use of anticonvulsants, especially given that typical anticonvulsant-induced side effects such as cognitive impairment, myelosuppression, liver dysfunction, and dermatological reactions appear to occur in patients with brain tumors more commonly than in other patient groups. After a review of the currently available literature, the American Academy of Neurology ${ }^{16}$ found that the administration of anticonvulsants does not offer enough benefit toward the prevention of first seizures to be used routinely in patients with brain tumors. Note, however, that anticonvulsants are commonly given to patients for a short time after craniotomy. If a patient has a documented seizure, it is important to consider the use of antiepileptic medications. If a patient is to undergo chemotherapy, it is important to watch for toxic interactions between these drug classes. ${ }^{40}$ Most of the pharmacokinetic interactions between these two drug classes are caused by their shared metabolism by the cytochrome P450 system in the liver. Different drugs cause either the induction or the inhibition of these enzymes. Levetiracetam does not cause the induction or inhibition of the P450 enzyme system or other enzyme systems, it has no active metabolite, and it exhibits almost no protein binding. These factors mean that this drug undergoes no significant interactions with other medications and appears suitable in patients requiring chemotherapy. ${ }^{40,44}$

\section{ConClusions}

In the management of brain metastasis there are several available treatment modalities. Authors of several studies have introduced the concept of individualizing treatment on the basis of prognostic factors. Tumor staging and grading are important, but the number of lesions is no longer an absolute factor in proceeding with surgery. Combining the modalities of surgery, WBRT, and radiosurgery has improved the outcome in patients with metastatic disease. Surgery continues to be a leading method in the treatment of brain metastases. With improvement in imaging and intraoperative guidance technologies, more tumors are now safely amenable to resection. With the development of new adjuncts such as BCNU wafers, surgical intervention can now be used with local intracranial therapy.

\section{References}

1. Agboola O, Benoit B, Cross P, Da Silva V, Esche B, Lesiuk H, et al: Prognostic factors derived from recursive partitioning analysis (RPA) of radiation therapy oncology group (RTOG) brain metastases trials applied to surgically resected and irradiated brain metastatic cases. Int J Radiat Oncol Biol Phys 42: $155-159,1998$

2. Arbit E, Wronski M, Burt M, Galicich JH: The treatment of patients with recurrent brain metastases. A retrospective analysis of 109 patients with nonsmall cell lung cancer. Cancer 76:765-773, 1995
3. Auchter RM, Lamond JP, Alexander E, Buatti JM, Chappell R, Friedman WA, et al: A multiinstitutional outcome and prognostic factor analysis of radiosurgery for resectable single brain metastasis. Int J Radiat Oncol Biol Phys 35:27-35, 1996

4. Bindal RK, Sawaya R, Leavens ME, Hess KR, Taylor SH: Reoperation for recurrent metastatic brain tumors. J Neurosurg 83:600-604, 1995

5. Bindal RK, Sawaya R, Leavens ME, Lee JJ: Surgical treatment of multiple brain metastases. J Neurosurg 79:210-216, 1993

6. Black PM: Solitary brain metastases. Radiation, resection, or radiosurgery? Chest 103 (4 Suppl):367S-369S, 1993

7. Brem S, Staller A, Wotoczek-Obadia M, Robb J, Vrionis FD, Pearlman JL, et al: Interstitial chemotherapy for local control of CNS metastases. Neuro-oncol 6:370-371, 2004 (Abstract)

8. Brown PD, Brown CA, Pollock BE, Gorman DA, Foote RL: Stereotactic radiosurgery for patients with "radioresistant" brain metastases. Neurosurgery 51:656-667, 2002

9. Delattre JY, Krol G, Thaler HT, Posner JB: Distribution of brain metastases. Arch Neurol 45:741-744, 1988

10. Eisner W, Burtscher J, Bale R, Sweeney R, Koppelstatter F, Golaszewski S, et al: Use of neuronavigation and electrophysiology in surgery of subcortically located lesions in the sensorimotor strip. J Neurol Neurosurg Psychiatry 72:378-381, 2002

11. Ewend MG, Brem S, Gilbert M, Goodkin R, Penar P: Treating single brain metastasis with resection, placement of BCNUpolymer wafers, and radiation therapy. AANS Abstract ID 13719, 2001

12. Ewend MG, Elbabaa S, Carey LA: Current treatment paradigms for the management of patients with brain metastases. Neurosurgery 57 (5 Suppl): S66-S77, 2005

13. Fife KM, Colman MH, Stevens GN, Firth IC, Moon D, Shannon KF, et al: Determinants of outcome in melanoma patients with cerebral metastases. J Clin Oncol 22:1293-1300, 2004

14. Gaspar L, Scott C, Rotman M, Asbell S, Phillips T, Wasserman I, et al: Recursive partitioning analysis (RPA) of prognostic factors in three Radiation Therapy Oncology Groups (RTOG) brain metastases trials. Int J Radiat Oncol Biol Phys 37: 745-751, 1997

15. Gavrilovic IT, Posner JB: Brain metastases: epidemiology and pathophysiology. J Neurooncol 75:5-14, 2005

16. Glantz MJ, Cole BF, Forsyth PA, Recht LD, Wen PY, Chamberlain MC, et al: Practice parameter: anticonvulsant prophylaxis in patients with newly diagnosed brain tumors. Report of the Quality Standards Subcommittee of the American Academy of Neurology. Neurology 54:1886-1893, 2000

17. Golden GA, Meldorf M, PROLONG Study Group: Patients with metastatic brain cancer undergoing resection and Gliadel implantation experienced low local recurrence rates in the PROLONG registry. Neuro-oncol 6:375-376, 2004 (Abstract)

18. Hu C, Chang EL, Hassenbusch SJ III, Allen PK, Woo SY, Mahajan A, et al: Nonsmall cell lung cancer presenting with synchronous solitary brain metastasis. Cancer 106:1998-2004, 2006

19. Largerwaard FJ, Levendag PC, Nowak PJ, Eijkenboom WM, Hanssens PE, Schmitz PI: Identification of prognostic factors in patients with brain metastases: a review of 1292 patients. Int J Radiat Oncol Biol Phys 43:795-803, 1999

20. McDermott MW, Sneed PK: Radiosurgery in metastatic brain cancer. Neurosurgery 57 (5 Suppl): S45-S53, 2005

21. Kondziolka D, Lunsford LD: Intraoperative navigation during resection of brain metastases. Neurosurg Clin N Am 7: 267-277, 1996

22. Kondziolka D, Lunsford LD, Flickinger JC: The radiobiology of radiosurgery. Neurosurg Clin N Am 10:157-166, 1999

23. Korinth MC, Delonge C, Hutter BO, Gilsbach JM: Prognostic factors for patients with microsurgically resected brain metastases. Onkologie 25:420-425, 2002

24. Patchell RA, Tibbs PA, Regine WF, Dempsey RJ, Mohiuddin 
M, Kryscio RJ, et al: Postoperative radiotherapy on the treatment of single metastases to the brain: a randomized trial. JAMA 280:1485-1489, 1998

25. Patchell RA, Tibbs PA, Walsh JW, Dempsey RJ, Maruyama Y, Kryscio RJ, et al: A randomized trial of surgery in the treatment of single metastases to the brain. N Engl J Med 322:494-500, 1990

26. Pollock BE, Brown PD, Foote RL, Stafford SL, Schomberg PJ: Properly selected patients with multiple brain metastases may benefit from aggressive treatment of their intracranial disease. J Neurooncol 61:73-80, 2003

27. Pruitt AA: Treatment of medical complications in patients with brain tumors. Curr Treat Options Neurol 7:323-336, 2005

28. Rao G, Klimo P Jr, Thompson CJ, Samlowski W, Wang M, Watson G, et al: Stereotactic radiosurgery as therapy for melanoma, renal carcinoma, and sarcoma brain metastases: impact of added surgical resection and whole brain radiotherapy. Int J Radiat Oncol Biol Phys 66 (4 Suppl):S20-S25, 2006

29. Sampson JH, Carter JH Jr, Friedman AH, Seigler HF: Demographics, prognosis, and therapy in 702 patients with brain metastases from malignant melanoma. J Neurosurg 88:11-20, 1998

30. Schiff D, DeAngelis LM: Therapy of venous thromboembolism in patients with brain metastases. Cancer 73:493-498, 1994

31. Schouten LJ, Rutten J, Huveneers HA, Twijnstra A: Incidence of brain metastases in a cohort of patients with carcinoma of the breast, colon, kidney, lung and melanoma. Cancer 94: 2698-2705, 2002

32. Selek U, Chang EL, Hassenbusch SJ III, Shiu AS, Lang FF, Allen P, et al: Stereotactic radiosurgical treatment in 103 patients for 153 cerebral melanoma metastases. Int J Radiat Oncol Biol Phys 59:1097-1106, 2004

33. Sheehan JP, Sun MH, Kondziolka D, Flickinger J, Lunsford LD: Radiosurgery in patients with renal cell carcinoma metastasis to the brain: long-term outcomes and prognostic factors influencing survival and local tumor control. J Neurosurg 98: 342-349, 2003

34. Sills AK: Current treatment approaches to surgery for brain metastases. Neurosurgery 57(5 Suppl): S24-S32, 2005

35. Sze G, Milano E, Johnson C, Heier L: Detection of brain metastases: comparison of contrast-enhanced MR with unenhanced MR and enhanced CT. AJNR Am J Neuroradiol 11:785-791, 1990
36. Tailibert S, Laigle-Donadey F, Chodkiewicz C, Sanson M, Hoang-Xuan K, Delattre JY: Leptomeningeal metastases from solid malignancy: a review. J Neurooncol 75:85-99, 2005

37. Tan TC, Black PM: Image-guided craniotomy for cerebral metastases: techniques and outcomes. Neurosurgery 53: 82-90, 2003

38. Unsgaard G, Selbekk T, Brostrup Muller T, Ommedal S, Torp SH, Myhr G, et al: Ability of navigated 3D ultrasound to delineate gliomas and metastases - comparison of image interpretations with histopathology. Acta Neurochir (Wien) 147: 1259-1269, 2005

39. Vecht CJ, Haaxma-Reiche H, Noordijk EM, Padberg GW, Voormolen JH, Hoekstra FH, et al: Treatment of single brain metastasis: radiotherapy alone or combined with neurosurgery? Ann Neurol 33:583-590, 1993

40. Vecht CJ, Wagner GL, Wilms EB: Interactions between antiepileptic and chemotherapeutic drugs. Lancet Neurol 2: 404-409, 2004

41. Vlieger EJ, Majoie CB, Leenstra S, Den Heeten GJ: Functional magnetic resonance imaging for neurosurgical planning in neurooncology. Eur Radiol 14:1143-1153, 2004

42. Vogelbaum MA, Suh JH: Resectable brain metastases. J Clin Oncol 24:1289-1294, 2006

43. Wadley J, Dorward N, Kitchen N, Thomas D: Pre-operative planning and intra-operative guidance in modern neurosurgery: a review of 300 cases. Ann R Coll Surg Engl 81:217-225, 1999

44. Wagner GL, Wilms EB, Van Donselaar CA, Vecht CJ: Levetiracetam: preliminary experience in patients with primary brain tumors. Seizure 12:585-586, 2003

45. Wronski M, Arbit E, McCormick B: Surgical treatment of 70 patients with brain metastases from breast carcinoma. Cancer 80:1746-1754, 1997

Manuscript submitted January 24, 2007.

Accepted February 7, 2007.

Address reprint requests to: Jonas M. Sheehan, M.D., Department of Neurosurgery, Pennsylvania State University, College of Medicine, Milton S. Hershey Medical Center, 500 University Drive, Hershey, Pennsylvania 17033. email: jsheehan@psu.edu. 